\title{
REJEITO DE MINERAÇÃo DE CARVÃO COMO ADSORVENTE PARA REMOÇÃo DA ACIDEZ, Fe (III), Al (III) E Mn (II) EM DRENAGEM ÁCIDA
}

\author{
Reginaldo Geremias* \\ Ciências Rurais, Universidade Federal de Santa Catarina, Campus Curitibanos, Rod. Ulisses Gaboardi, km 3, s/n, 89520-000 \\ Curitibanos - SC, Brasil \\ Rogério Laus e Valfredo Tadeu de Fávere \\ Departamento de Química, Universidade Federal de Santa Catarina, CP 476, 88040-900 Florianópolis - SC, Brasil \\ Rozangela Curi Pedrosa \\ Departamento de Bioquímica, Universidade Federal de Santa Catarina, CP 476, 88040-900 Florianópolis - SC, Brasil
}

Recebido em 4/11/09; aceito em 29/4/10; publicado na web em 20/7/10

\begin{abstract}
COAL MINING WASTE AS ADSORBENT FOR REMOVAL OF ACIDITY, Fe (III), Al (III) and Mn (II) IN ACID DRAINAGE. This study aimed the use of coal mining waste as a new adsorbent for $\mathrm{H}_{3} \mathrm{O}^{+}$and removal of $\mathrm{Al}$ (III), Fe (III) and Mn (II) from acid mine drainage. Data from kinetic and equilibrium of the adsorption of $\mathrm{H}_{3} \mathrm{O}^{+}$followed the pseudo second-order and Langmuir isotherm models. The maximum adsorption capacity of $\mathrm{H}_{3} \mathrm{O}^{+}$was $316 \mathrm{mmol} \mathrm{kg}^{-1}$. The adsorbent removed $100 \%$ of $\mathrm{Al}$ (III), $100 \%$ of $\mathrm{Fe}$ (III) and $89 \%$ of Mn (II), suggesting its use as an alternative for the treatment of acid mine drainage.
\end{abstract}

Keywords: coal mining waste; adsorption $\mathrm{H}_{3} \mathrm{O}^{+}$; removal metallic ions.

\section{INTRODUÇÃO}

A extração e beneficiamento de carvão mineral é uma importante atividade econômica da região sul de Santa Catarina, Brasil. Entretanto, neste processo são geradas drenagens ácidas de mina (DAM) caracterizadas pela expressiva acidez $(\mathrm{pH}<3,0)$ e elevada concentração de íons metálicos (ex: Al (III), Fe (III), Mn (II), Zn (II), $\mathrm{Cu}$ (II), Cd (II) e Pb (II)), sendo capazes de atingir os mananciais hídricos. ${ }^{1,2}$ Tem-se proposto que ambiente aquático cujo $\mathrm{pH}$ e as concentrações de íons metálicos se encontram em níveis não tolerados pode causar efeitos deletérios e, até mesmo, a morte da biota exposta. ${ }^{3-6}$

Além da drenagem ácida, a geração de rejeitos de mineração também se constitui em um dos principais impactos decorrentes da atividade carbonífera. Dentre as alterações ambientais promovidas pelos rejeitos pode-se citar a supressão de áreas de vegetação, reconfiguração de superfícies topográficas, impacto visual, aceleração de processos erosivos, inviabilidade de uso alternativo do solo, emissão de gases e partículas no ar, aumento da turbidez e assoreamento de corpos d'água, geração de drenagem ácida, contaminação dos recursos hídricos e comprometimento da qualidade de vida da população do seu entorno. ${ }^{7-9}$ Desta forma, a busca de alternativas de tratamento destes efluentes, bem como, do aproveitamento econômico dos rejeitos é de suma importância para minimizar os seus efeitos sobre o meio ambiente.

Diversos tipos de tratamento de drenagem ácida têm sido propostos e, para tanto, são levados em consideração os volumes gerados, análises qualitativa e quantitativa dos contaminantes presentes, capacidade de reduzir a sua acidez, bem como, os níveis de íons metálicos do meio. ${ }^{10}$ Em geral, o tratamento destes efluentes é feito através de uma pré-correção de $\mathrm{pH}$, com posterior utilização de resinas iônicas, colunas de filtração, processos de adsorção, coagulação, floculação e flotação para remoção de íons metálicos. Contudo, estes processos demandam elevados recursos financeiros, fazendo-se necessária a busca de novas tecnologias de

*e-mail: reginaldogeremias@gmail.com tratamento, utilizando-se materiais de baixo custo e com melhor capacidade de remoção dos poluentes. ${ }^{11,12}$

Neste contexto, a utilização de óxidos de metais obtidos por processo de calcinação de rejeito de mineração de carvão poderia ser uma alternativa de tratamento das drenagens. Estes óxidos seriam capazes de atuar como adsorventes de $\mathrm{H}_{3} \mathrm{O}^{+}$, provocando a elevação do $\mathrm{pH}$ com consequente precipitação e remoção de íons metálicos em solução, além de adsorvê-los por atração eletrostática e forças dipolo-dipolo, em decorrência da presença de cargas superficiais. ${ }^{13,14}$

Entretanto, para uma melhor avaliação da capacidade de adsorção pelo adsorvente em questão, são necessários estudos dos fatores que influenciam neste processo, podendo-se citar o efeito da temperatura, o tempo de contato para o sistema alcançar o equilíbrio, a concentração dos íons, a quantidade de adsorvente, a quantidade adsorvida em função da concentração, os quais podem ser descritos através de modelos cinéticos e de isotermas. ${ }^{15,16}$

Partindo destes pressupostos, o presente trabalho teve como objetivo utilizar rejeito de mineração de carvão como adsorvente para íons $\mathrm{H}_{3} \mathrm{O}^{+}$e na remoção de íons metálicos, com vistas a sua aplicação como alternativa de tratamento de drenagem ácida de mina, conferindo uma utilidade econômica ao rejeito.

\section{PARTE EXPERIMENTAL}

\section{Drenagem ácida}

As amostras de drenagem ácida de mina de carvão com pH 2,6 foram coletadas em frascos de polietileno $(50 \mathrm{~L})$ em uma empresa de mineração de carvão de subsolo situada no município de Criciúma/ $\mathrm{SC}$ - Brasil. As concentrações dos íons metálicos Al (III), Fe (III), $\mathrm{Mn}$ (II), Zn (II), Cu (II), Cd (II) e Pb (II) foram determinadas por espectrometria de absorção atômica em chama (FAAS), empregando espectrômetro Hitachi modelo Z8230 equipado com corretor de fundo por efeito Zeeman, atomizador ar-acetileno e óxido nitroso-acetileno e lâmpada de cátodo oco específica para os íons metálicos e espectrômetro Varian modelo Spectraa 50. 


\section{Preparação do adsorvente}

Rejeitos de mineração de carvão depositados na superfície do solo foram coletados em companhia mineradora da região carbonífera do sul de Santa Catarina - Brasil. As amostras foram tamizadas em peneiras de 40 mesh para a obtenção de granulometria mais homogênea. $\mathrm{O}$ rejeito foi submetido à calcinação por $1 \mathrm{~h}$ à $800{ }^{\circ} \mathrm{C}$ para a obtenção dos óxidos de metais a serem utilizados como material adsorvente, utilizando-se Mufla Jung modelo Digimec BTC 9090. Posteriormente, foi efetuada a caracterização dos óxidos de metais obtidos, utilizando-se difratômetro Philips modelo X' Pert, com radiação $\mathrm{CuK} \alpha \mathrm{l}(\lambda=1,54056 \AA$ ) e gerador de raio-X operado em 40 $\mathrm{kV}$ e $30 \mathrm{~mA}$. Constatou-se a presença majoritária dos óxidos $\mathrm{SiO}_{2}$, $\mathrm{Al}_{2} \mathrm{O}_{3}$ e $\mathrm{Fe}_{2} \mathrm{O}_{3}$ no material adsorvente. ${ }^{14}$

\section{Experimentos de adsorção de $\mathrm{H}_{3} \mathrm{O}^{+}$}

Todos os experimentos de adsorção foram realizados em banho termostatizado à $25^{\circ} \mathrm{C}$ com agitação de $250 \mathrm{rpm}$ em incubadora mini shaker Marconi modelo MA 832. A concentração dos íons $\mathrm{H}_{3} \mathrm{O}^{+}$foi determinada por potenciometria em pHmetro Corning modelo $\mathrm{pH} /$ ION Analyzer 350.

\section{Cinética de adsorção}

As cinéticas de adsorção de $\mathrm{H}_{3} \mathrm{O}^{+}$foram realizadas em frascos fechados contendo volume fixo de drenagem ácida de mina $(50 \mathrm{~mL})$ e diferentes quantidades de adsorvente $(0,3 ; 0,5$ e 1,0 g). As amostras foram mantidas sob agitação, sendo o $\mathrm{pH}$ monitorado em diferentes intervalos de tempo $(0,5 ; 1 ; 3 ; 6 ; 10 ; 24$ e $48 \mathrm{~h})$ para posterior avaliação do equilíbrio de adsorção.

\section{Isoterma de adsorção}

Nos ensaios de equilíbrio de adsorção de $\mathrm{H}_{3} \mathrm{O}^{+}$foram utilizados frascos fechados contendo $25 \mathrm{~mL}$ de drenagem ácida de mina em contato com diferentes massas de adsorvente $(0,1 \mathrm{a} 2,0 \mathrm{~g})$. Após atingir o equilíbrio de adsorção, determinou-se o $\mathrm{pH}$ por potenciometria para posterior avaliação da quantidade de $\mathrm{H}_{3} \mathrm{O}^{+}$adsorvida.

\section{Experimentos de remoção de íons metálicos}

Diferentes massas de rejeito de mineração de carvão calcinado $(0,3 ; 0,5 ; 1,0$ e $2,0 \mathrm{~g})$ foram colocadas em contato com $50 \mathrm{~mL}$ de drenagem em erlenmeyers, por 48 h, sob agitação (200 rpm) e à temperatura ambiente. Posteriormente, o material foi filtrado, sendo determinado o $\mathrm{pH}$ por potenciometria e as concentrações dos metais majoritários por FAAS para a avaliação do percentual de remoção dos íons em solução.

\section{RESULTADOS E DISCUSSÃO}

\section{Cinética de adsorção de $\mathrm{H}_{3} \mathrm{O}^{+}$}

Para avaliar o mecanismo cinético que controla o processo de adsorção, foram empregados os modelos de pseudoprimeira ordem (Equação 1) e pseudossegunda ordem (Equação 2), onde $k_{1}$ e k são as constantes de velocidade da adsorção de pseudoprimeira ordem e pseudossegunda ordem, respectivamente, $\mathrm{q}_{\mathrm{t}}$ é a quantidade adsorvida no tempo t e $\mathrm{q}_{\mathrm{e}}$ é a quantidade adsorvida no equilíbrio. A velocidade inicial de adsorção $\left(h_{\mathrm{o}}\right)$ foi determinada através dos valores de $\mathrm{q}_{\mathrm{e}} \mathrm{e}$ $\mathrm{k}_{2}$, empregando a Equação 3. A validade desses modelos foi interpretada pela linearidade dos gráficos $\log (\mathrm{qe}-\mathrm{qt}) v s$. $\mathrm{t}$ e (t/qt) vs. $\mathrm{t}$, respectivamente. ${ }^{17}$

$$
\begin{gathered}
\log \left(\mathrm{q}_{\mathrm{e}}-\mathrm{q}_{\mathrm{t}}\right)=\log \mathrm{q}_{\mathrm{e}}-\frac{\mathrm{k}_{1}}{2,303} \mathrm{t} \\
\frac{\mathrm{t}}{\mathrm{q}_{\mathrm{t}}}=\frac{1}{\mathrm{k}_{2} \mathrm{q}_{\mathrm{e}}^{2}}+\frac{1}{\mathrm{q}_{\mathrm{e}}} \mathrm{t} \\
\mathrm{h}_{0}=\mathrm{k}_{2} \mathrm{q}_{\mathrm{e}}^{2}
\end{gathered}
$$

A Figura 1 apresenta a cinética de adsorção de íons $\mathrm{H}_{3} \mathrm{O}^{+}$da drenagem ácida pelo adsorvente, utilizando-se diferentes massas. $\mathrm{O}$ perfil cinético permite demonstrar que houve expressiva adsorção nas primeiras $3 \mathrm{~h}$ de contato para as diferentes massas, sendo que o equilíbrio foi atingido mais rapidamente com 1,0 e $0,5 \mathrm{~g}(3 \mathrm{~h})$ e necessitando de maior tempo com $0,3 \mathrm{~g}(6 \mathrm{~h})$. Isto também pode ser constatado nos valores de $\mathrm{k}_{2}$ e $\mathrm{h}_{\mathrm{o}}$ obtidos nos parâmetros cinéticos.

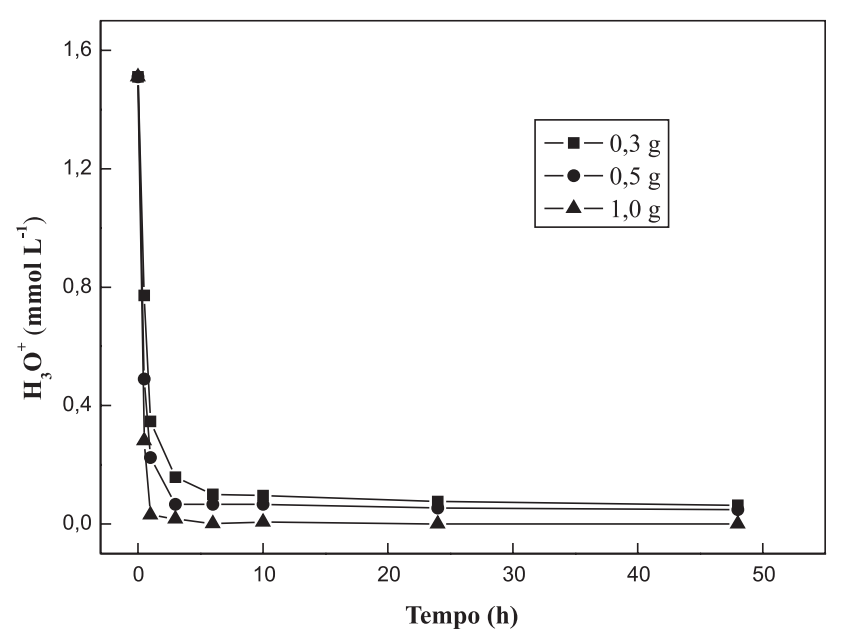

Figura 1. Variação da concentração de $\mathrm{H}_{3} \mathrm{O}^{+}$adsorvido em função do tempo pelo adsorvente. $\left[\mathrm{H}_{3} \mathrm{O}^{+}\right]_{\text {inicial }}=1,51 \mathrm{mmol} \mathrm{L}^{-1}$; temperatura $=25^{\circ} \mathrm{C}$; velocidade de agitação $=250 \mathrm{rpm}$

As equações lineares e os respectivos coeficientes de correlação obtidos na adsorção de $\mathrm{H}_{3} \mathrm{O}^{+}$estão descritos na Tabela 1. A análise dos resultados permitiu verificar que a equação de pseudossegunda ordem foi a que forneceu o melhor ajuste dos dados experimentais para os ensaios com diferentes massas de adsorvente, sugerindo ser o modelo mais apropriado para a interpretação do mecanismo de adsorção. Os resultados mostraram que a velocidade de adsorção depende da quantidade do íon na superfície do adsorvente e da quantidade adsorvida no equilíbrio.

Tabela 1. Equações lineares e respectivos coeficientes de correlação (R) de adsorção de $\mathrm{H}_{3} \mathrm{O}^{+}$

\begin{tabular}{lcccc}
\hline Massa (g) & \multicolumn{2}{c}{ Pseudoprimeira ordem } & \multicolumn{2}{c}{ Pseudossegunda ordem } \\
& Equação & $\mathrm{R}$ & Equação & $\mathrm{R}$ \\
\hline 0,3 & $\mathrm{Y}=1,418+0,061 \mathrm{X}$ & 0,845 & $\mathrm{Y}=1,171+4,114 \mathrm{X}$ & 0,999 \\
0,5 & $\mathrm{Y}=1,971+0,067 \mathrm{X}$ & 0,728 & $\mathrm{Y}=1,153+6,803 \mathrm{X}$ & 0,999 \\
1,0 & $\mathrm{Y}=2,775+0,068 \mathrm{X}$ & 0,686 & $\mathrm{Y}=0,576+13,194 \mathrm{X}$ & 1,000 \\
\hline
\end{tabular}

A partir do tratamento cinético de adsorção de $\mathrm{H}_{3} \mathrm{O}^{+}$pelo adsorvente (Figura 2), obtiveram-se os parâmetros cinéticos (Tabela 2), onde os resultados permitem demonstrar que os valores de $\mathrm{q}_{\mathrm{e}}$ calculados pelo modelo da equação de pseudossegunda ordem estão em concordância com os valores de $\mathrm{q}_{\mathrm{e}}$ experimentais. Entretanto, para o modelo de pseudoprimeira ordem, constata-se uma expressiva discrepância entre os seus valores nas diferentes massas de adsorvente, 
obtendo-se erros relativos de 75, 93 e 77\% para 0,3; 0,5 e 1,0 g, respectivamente. Constata-se também que a constante de velocidade $\left(\mathrm{k}_{2}\right)$ e a velocidade de adsorção inicial $\left(h_{\mathrm{o}}\right)$ foram dependentes da massa do adsorvente, sendo mais significativa com a utilização de $1,0 \mathrm{~g}$ de adsorvente, cujo perfil é constatado na curva cinética.

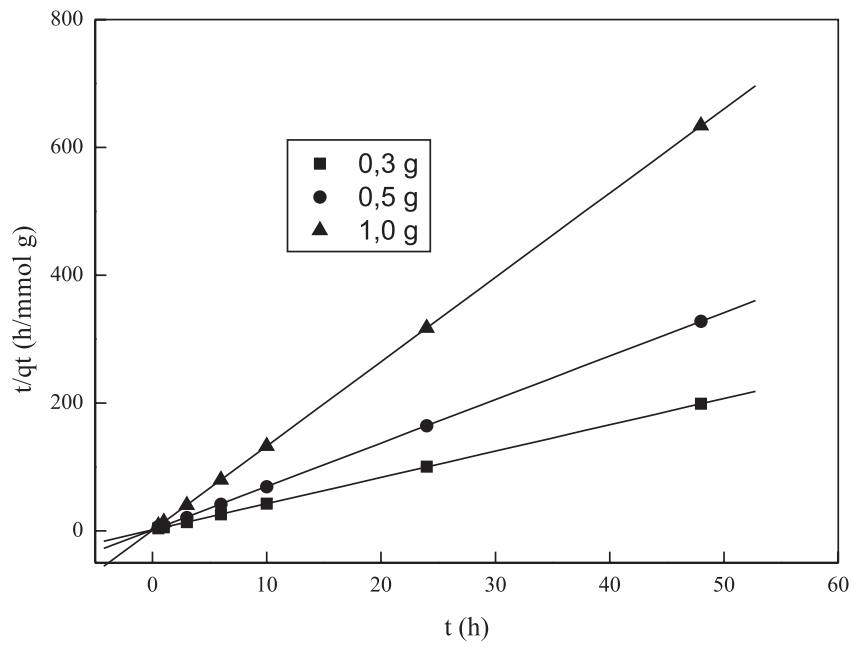

Figura 2. Linearização da equação de pseudossegunda ordem de adsorção de $\mathrm{H}_{3} \mathrm{O}^{+}$

Tabela 2. Parâmetros cinéticos de adsorção de $\mathrm{H}_{3} \mathrm{O}^{+}$

\begin{tabular}{lccccccc}
\hline & \multicolumn{3}{c}{ Pseudoprimeira ordem } & \multicolumn{4}{c}{ Pseudossegunda ordem } \\
$\begin{array}{l}\text { Massa } \\
(\mathrm{g})\end{array}$ & $\begin{array}{c}\mathrm{q}_{\mathrm{e}} \text { exp. } \\
\left(\mathrm{mmol} \mathrm{g}^{-1}\right)\end{array}$ & $\begin{array}{c}\mathrm{q}_{\mathrm{e}} \text { calc. } \\
\left(\mathrm{mmol} \mathrm{g}^{-1}\right)\end{array}$ & $\begin{array}{c}\mathrm{k}_{1} \\
\left(\mathrm{~h}^{-1}\right)\end{array}$ & $\begin{array}{c}\mathrm{q}_{\mathrm{e}} \text { exp. } \\
\left(\mathrm{mmol} \mathrm{g}^{-1}\right)\end{array}$ & $\begin{array}{c}\mathrm{q}_{\mathrm{e}} \text { calc. } \\
\left(\mathrm{mmol} \mathrm{g}^{-1}\right)\end{array}$ & $\begin{array}{c}\mathrm{k}_{2} \\
\mathrm{mmol}^{-1} \\
\left.\mathrm{~h}^{-1}\right)\end{array}$ & $\begin{array}{c}\mathrm{h}_{\mathrm{o}} \\
\left(\mathrm{mmol}^{-1}\right. \\
\mathrm{g}^{-1} \mathrm{~h}^{-1}\end{array}$ \\
\hline 0,3 & 0,241 & 0,038 & 0,141 & 0,241 & 0,243 & 14,45 & 0,84 \\
0,5 & 0,146 & 0,010 & 0,155 & 0,146 & 0,147 & 40,16 & 0,86 \\
1,0 & 0,075 & 0,001 & 0,157 & 0,075 & 0,075 & 303 & 1,74 \\
\hline
\end{tabular}

\section{Isoterma de adsorção de $\mathrm{H}_{3} \mathrm{O}^{+}$}

Nos estudos de isoterma de adsorção, a quantidade adsorvida de $\mathrm{H}_{3} \mathrm{O}^{+}$foi obtida empregando a Equação 4 onde $\mathrm{q}_{\mathrm{e}}$ é quantidade de íons adsorvido no equilíbrio ( $\left.\mathrm{mmol} \mathrm{g}^{-1}\right), \mathrm{C}_{\mathrm{i}}$ é a concentração inicial de íons em solução $\left(\mathrm{mmol} \mathrm{L}^{-1}\right), \mathrm{C}_{\mathrm{e}}$ é a concentração de íons em solução no equilíbrio ( $\left.\mathrm{mmol} \mathrm{L}^{-1}\right), \mathrm{V}$ é o volume da solução $(\mathrm{L})$ e m é a massa do adsorvente $(\mathrm{g}):^{18}$

$$
\mathrm{q}_{\mathrm{e}}=\frac{\left(\mathrm{C}_{\mathrm{i}}-\mathrm{C}_{\mathrm{e}}\right) \mathrm{V}}{\mathrm{m}}
$$

Para a interpretação dos dados de adsorção, foram testados os modelos de isoterma de Langmuir (Equação 5) e de Freundlich (Equação 6), onde $\mathrm{q}_{\mathrm{m}}$ é a quantidade máxima de íons adsorvidos, $\mathrm{C}_{\mathrm{e}}$ é a concentração de íons em solução no equilíbrio, $\mathrm{K}_{\mathrm{ads}}\left(\mathrm{L} \mathrm{mg}^{-1}\right)$ é a constante de equilíbrio de adsorção, $\mathrm{K}_{\mathrm{F}}\left[\left(\mathrm{mg} \mathrm{g}^{-1}\right)\left(\mathrm{L} \mathrm{mg}^{-1}\right)^{1 / n}\right]$ é a constante de Freundlich e $b_{\mathrm{F}}$ (adimensional) é o fator que indica a heterogeneidade da superfície do adsorvente. ${ }^{18}$

$$
\begin{gathered}
\mathrm{q}_{\mathrm{e}}=\frac{\mathrm{q}_{\mathrm{m}} \mathrm{K}_{\mathrm{ads}} \mathrm{C}_{\mathrm{e}}}{1+\mathrm{K}_{\mathrm{ads}} \mathrm{C}_{\mathrm{e}}} \\
\mathrm{q}_{\mathrm{e}}=\mathrm{K}_{\mathrm{F}} \mathrm{C}_{\mathrm{e}}^{\mathrm{b}_{\mathrm{F}}}
\end{gathered}
$$

No modelo de Langmuir, os sítios de adsorção são todos de mesma energia, porém, em muitos casos, a adsorção pode não se ajustar a este modelo de isoterma. Em algumas situações, a isoterma de Freundlich proporciona melhores ajustes dos dados experimentais. ${ }^{18} \mathrm{~A}$ isoterma de Freundlich descreve o equilíbrio em superfícies heterogêneas e, por esta razão, não assume uma capacidade de adsorção em monocamada.

A isoterma de equilíbrio de adsorção de íons $\mathrm{H}_{3} \mathrm{O}^{+}$na drenagem ácida pelo adsorvente está ilustrada na Figura 3. Pode-se observar a correlação entre a quantidade de íon adsorvida na superfície do adsorvente e a concentração de íon remanescente na fase aquosa em equilíbrio. Esta relação mostrou que a quantidade adsorvida aumentou com a concentração de equilíbrio do íon na fase aquosa, alcançando progressivamente a saturação do adsorvente.

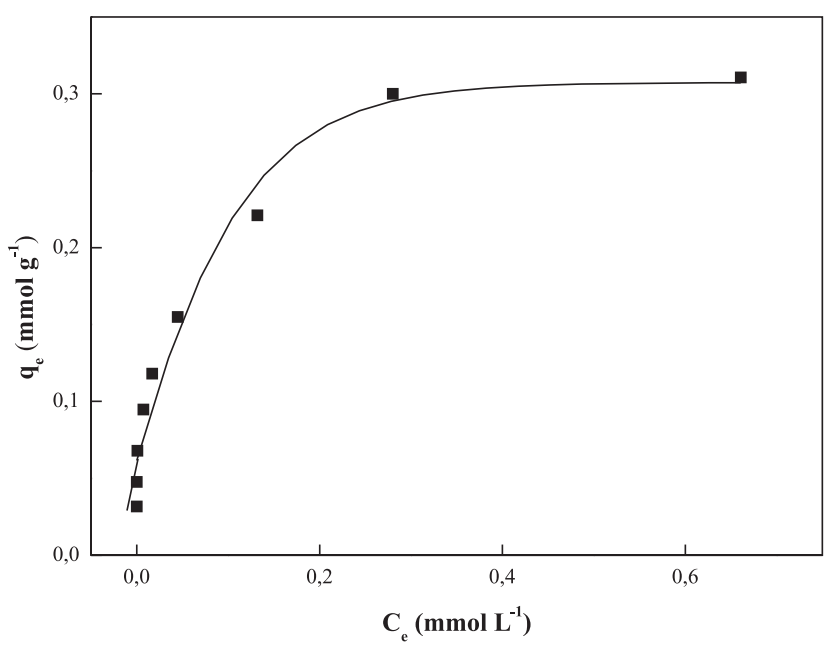

Figura 3. Isoterma de equilíbrio de adsorção dos íons $\mathrm{H}_{3} \mathrm{O}^{+}$. $\left[\mathrm{H}_{3} \mathrm{O}^{+}\right]_{\text {inicial }}=1,51$ mmol $L^{-1}$; volume de amostra $=25 \mathrm{~mL}$; variação da massa de adsorvente 0,100-2,00 g; tempo de contato $=24 \mathrm{~h}$; temperatura $=25^{\circ} \mathrm{C}$; velocidade de agitação $=250 \mathrm{rpm}$

Na interpretação dos dados de adsorção de $\mathrm{H}_{3} \mathrm{O}^{+}$, observou-se que o modelo de isoterma de Langmuir apresentou melhor ajuste dos dados experimentais de equilíbrio, o que pode ser observado a partir do valor do coeficiente de correlação $(\mathrm{R}=0,995)$ obtido pela linearização da equação, quando comparado ao da isoterma de Freundlich $(R=0,966)$. Além disso, os valores teóricos das quantidades adsorvidas calculados a partir da equação de Langmuir forneceram um erro relativo de $17 \%$, enquanto a equação de Freundlich foi de $20 \%$. Este perfil sugere que a superfície do adsorvente tem características mais homogêneas e com sítios idênticos de energia, evidenciando, portanto, uma adsorção que ocorre em monocamada. Os resultados indicam uma capacidade máxima de adsorção de $0,316 \mathrm{mmol} \mathrm{H}_{3} \mathrm{O}^{+} / \mathrm{g}$ de adsorvente $(316 \mathrm{mmol} / \mathrm{kg}$ ).

Portanto, nossos resultados permitem constatar que o adsorvente obtido a partir da calcinação do rejeito de mineração de carvão foi capaz de promover a adsorção de $\mathrm{H}_{3} \mathrm{O}^{+}$. Esta capacidade de adsorção poderia estar relacionada à presença dos óxidos $\mathrm{SiO}_{2}, \mathrm{Al}_{2} \mathrm{O}_{3}$ e $\mathrm{Fe}_{2} \mathrm{O}_{3}$ encontrados na caracterização do material adsorvente, uma vez que se tem sugerido que óxidos de metais, quando em meio aquoso, apresentam as superfícies recobertas por camadas de grupos hidroxil, que possibilitam a ocorrência de equilíbrios de adsorção, destacando-se equilíbrio ácido-base (Equações 7 e 8). ${ }^{19-22}$

$$
\begin{gathered}
\mathrm{S}-\mathrm{OH}+\mathrm{H}_{3} \mathrm{O}^{+} \rightleftarrows \mathrm{S}-\mathrm{OH}_{2}^{+}+\mathrm{H}_{2} \mathrm{O} \\
\mathrm{S}-\mathrm{OH}+\mathrm{OH}^{-} \rightleftarrows \mathrm{S}-\mathrm{O}^{-}+\mathrm{H}_{2} \mathrm{O}
\end{gathered}
$$

O grupo hidroxil exibe um comportamento como base de Lewis e, portanto, a adsorção dos íons $\mathrm{H}_{3} \mathrm{O}^{+}$envolve a protonação dos grupos hidroxil da superfície do adsorvente. 


\section{Remoção de metais}

A Tabela 3 apresenta os resultados da concentração dos metais na drenagem ácida, onde se constata a presença majoritária de $\mathrm{Al}$ (III), Fe (III) e Mn (II), conforme também tem sido evidenciado na literatura. ${ }^{6,23,24}$ Este perfil revela que as concentrações dos íons metálicos Fe (III), Al (III) e Mn (II) estão acima dos preconizados pela Resolução $n^{\circ} 397$ do CONAMA para as condições de lançamentos de efluentes. ${ }^{25}$ Desta forma, a drenagem se mostra potencialmente tóxica, havendo a necessidade de seu tratamento antes de ser lançadas ao meio ambiente.

Tabela 3. Concentração de metais na drenagem ácida e respectivos valores máximos previstos pela Resolução no 397 do CONAMA

\begin{tabular}{lcc}
\hline Íons metálicos & $\begin{array}{c}\text { Concentração drenagem } \\
\left(\mathrm{mg} \mathrm{L}^{-1}\right)\end{array}$ & $\begin{array}{c}\text { Concentração resolução } \\
\left(\mathrm{mg} \mathrm{L}^{-1}\right)\end{array}$ \\
\hline $\mathrm{Al}$ (III) & 30,6 & $* \mathrm{NP}$ \\
$\mathrm{Fe}$ (III) & 15,8 & 15,0 \\
$\mathrm{Mn}$ (II) & 11,4 & 1,0 \\
$\mathrm{Zn}$ (II) & 1,2 & 5,0 \\
$\mathrm{Cu}$ (II) & 0,2 & 1,0 \\
$\mathrm{Cd}$ (II) & 0,01 & 0,2 \\
$\mathrm{~Pb}$ (II) & $* * \mathrm{ND}$ & 0,5 \\
\hline
\end{tabular}

* NP = não previsto; $* * \mathrm{ND}=$ não detectado

Para a avaliação do uso do rejeito de mineração como material alternativo no tratamento da drenagem ácida foram efetuados estudos de remoção dos íons metálicos majoritários $\mathrm{Al}$ (III), Fe (III) e Mn (II), cujos resultados estão demonstrados na Figura 4. Pode-se observar que o uso de $1,0 \mathrm{~g}$ do rejeito calcinado foi suficiente para promover completa remoção dos íons Al (III) e Fe (III), sendo que a remoção de Mn (II) foi mais efetiva no tratamento com 2,0 g.

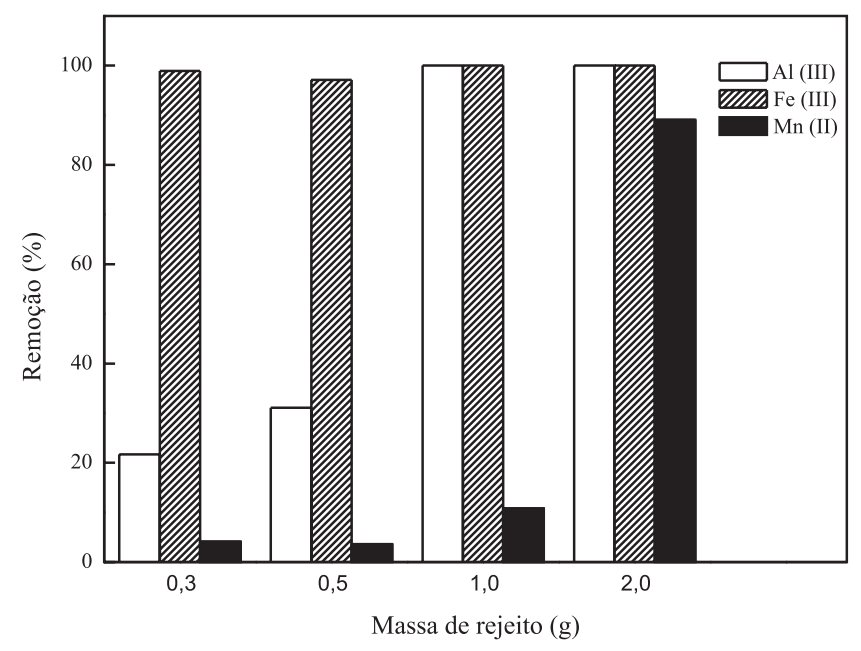

Figura 4. Remoção de metais em drenagem ácida tratada com diferentes massas de rejeito calcinado

A capacidade de remoção dos íons metálicos poderia ser decorrente da presença dos óxidos de metais obtidos por calcinação do rejeito, os quais seriam capazes de promover a adsorção dos íons $\mathrm{H}_{3} \mathrm{O}^{+}$do meio, o que conduziria à elevação do $\mathrm{pH}$, com consequente formação e precipitação de óxido e/ou hidróxidos insolúveis de Al (III), Fe (III) e Mn (II). Também é possível que os íons metálicos estejam sendo removidos por adsorção aos óxidos presentes no rejeito, através de interação por complexação, formação de par iônico, troca iônica, interação eletrostática, forças de van der Walls, entre outros mecanismos. ${ }^{26}$

Os valores de $\mathrm{pH}$ obtidos após o tratamento da drenagem com diferentes massas do rejeito foram 4,2 (0,3 g), 4,3 (0,5 g), 6,5 (1,0 g) e 7,8 (2,0 g). Tem-se proposto que em valores de $\mathrm{pH}$ próximos a 3,7 e 5,4 ocorre, respectivamente, a precipitação do ferro e alumínio na forma dos hidróxidos $\mathrm{Fe}(\mathrm{OH})_{3}$ e $\mathrm{Al}(\mathrm{OH})_{3}$, sendo que a precipitação do manganês, na forma de $\mathrm{Mn}(\mathrm{OH})_{2}$, ocorre somente em $\mathrm{pH}$ superior a $11,0 .{ }^{27}$ Este comportamento, portanto, poderia justificar a maior efetividade de remoção dos íons metálicos Fe (III) e Al (III), quando comparado ao Mn (II), conforme observado em nossos resultados.

A partir de todos os resultados obtidos, pode-se sugerir o uso de rejeitos de mineração de carvão no tratamento de drenagem ácida, uma vez que se mostraram capazes de adsorver $\mathrm{H}_{3} \mathrm{O}^{+}$e promover a remoção de metais comumente presentes nestes efluentes, minimizando os seus impactos sobre o meio ambiente.

\section{CONCLUSÃO}

Os estudos indicaram que o rejeito de mineração calcinado foi capaz de promover a adsorção de $\mathrm{H}_{3} \mathrm{O}^{+}$em drenagem ácida. $\mathrm{O}$ modelo de pseudossegunda ordem foi o que melhor correlacionou os dados cinéticos experimentais, sugerindo ser o modelo mais apropriado para a interpretação do mecanismo de adsorção, e que a velocidade de adsorção é dependente das quantidades do íon na superfície do adsorvente e adsorvido no equilíbrio. Nos estudos de equilíbrio de adsorção, constatou-se que o modelo de Langmuir foi o que melhor se ajustou aos dados experimentais, sugerindo que a superfície do adsorvente é homogênea e com sítios idênticos de energia, e evidenciando uma adsorção em monocamada. O rejeito calcinado promoveu expressiva remoção dos íons metálicos majoritários presentes na drenagem, sugerindo seu uso como uma alternativa de tratamento destas fontes de contaminação ambiental.

\section{AGRADECIMENTOS}

À Universidade Federal de Santa Catarina, aos laboratórios QUITECH e LABIOEX e ao Conselho Nacional de Desenvolvimento Científico e Tecnológico (CNPq) pelo apoio financeiro.

\section{REFERÊNCIAS}

1. Lattuada, R. M.; Menezes, C. T. B.; Pavei, P. T.; Peralba, M. C. R.; Dos Santos, J. H. Z.; J. Hazard. Mater. 2009, 163, 531.

2. Wei, X.; Viadero, R. C. Jr; Bhojappa, S.; Water Res. 2008, 42, 3275.

3. Ridge, T. Em Coal Mine Drainage Prediction an Pollution Prevention in Pennsylvania; Ridge, T.; Seif, J. M., eds.; Pennsylvania Department of Environmental Protection: Pennsylvania, 1998, cap. 4.

4. Benassi, J. C.; Laus, R.; Geremias, R.; Lima, P. L.; Menezes, C. T. B.; Laranjeira, M. C. M.; Wilhelm-Filho, D.; Fávere, V. T.; Pedrosa, R. C.; Arch. Environ. Contam. Toxicol. 2006, 51, 633.

5. Maccausland, A.; McTammany, M. E.; Environ. Pollut. 2007, 149, 216.

6. Martins, N.; Bollinger, C.; Harper, R. M.; Ribeiro, R.; Aquat. Toxicol. 2009, 92, 104.

7. Komnitsas, K.; Kontopoulos, A.; Lazar, I.; Cambridge, M.; Miner. Eng. 1998, $1,1179$.

8. Qiang, X.; Bing, L.; Hui-yun, W.; Lei, L.; J. Trace Elem. Med. Biol. 2006, 20, 97.

9. Geremias, R.; Tese de Doutorado, Universidade Federal de Santa Catarina, Brasil, 2008.

10. Gasea, B.; Adam, K.; Kontopoulos, A.; Miner. Eng. 1996, 9, 23.

11. van Rensburg, L.; Morgenthal, T. L.; J. Environ. Qual. 2003, 32, 1658.

12. Johnson, D. B.; Hallberg, K. B.; Sci. Total Environ. 2005, 338, 3. 
13. Babel, S.; Kurniawan, T. A.; J. Hazard. Mater. 2003, 97, 219.

14. Geremias, R.; Laus, R.; Macan, J. M.; Pedrosa, R. C.; Laranjeira, M. C. M.; Silvano, J.; Fávere, V. T.; Environ. Technol. 2008, 29, 863.

15. Vitali, L.; Laranjeira, M. C. M.; Fávere, V. T.; Quim. Nova 2008, 31, 1400.

16. Mishra, P. C.; Patel, R. K.; J. Hazard. Mater. 2009, 168, 319.

17. Wu, F.; Tseng, R.; Juang, R.; Water Res. 2001, 35, 613.

18. Justi, K. C.; Tese de Doutorado, Universidade Federal de Santa Catarina, Brasil, 2006.

19. Dzonbak, D. A.; Morel, F. M. M.; Surface Complexation Modeling: Hydrons Ferric Oxide, $1^{\text {st }}$ ed., Wiley-Interscience: New York, 1990.

20. Nano, G. V.; Strathmann, T. J.; J. Colloid Interface Sci. 2006, 297, 443.
21. Ríos, C. A.; Williams, C. D.; Roberts, C. L.; J. Hazard. Mater. 2008 , $156,23$.

22. Wang, P.; Lo, I. M.; Water Res. 2009, 43, 3727.

23. Laus, R.; Laranjeira, M. C. M.; Martins, A. O.; Fávere, V. T.; Benassi, J. C.; Pedrosa, R. C.; Geremias, R.; Quim. Nova 2006, 29, 34.

24. Campaner, V. P.; Luiz-Silva, W.; Quim. Nova 2009, 32, 146.

25. http://www.mma.gov.br/port/conama/, acessada em Março 2010.

26. Wu, C. H.; Lin, C. F.; Chen, W. R.; J. Environ. Sci. Health, Part A: Toxic/ Hazard. Subst. Environ. Eng. 2004, 39, 717.

27. Marti, F. B.; Química Analitica Cualitativa,16 ed., Paraninfo: Madri, 1998 